\title{
Fronto-orbital intradiploic transitional meningioma
}

Sir,

Primary intraosseous or ectopic meningioma of the skull is a rare tumor accounting for about $1 \%$ of meningiomas. These arise in paranasal sinuses, neck, orbit and very rarely in diploe of cranial bones. Intradiploic meningioma of the orbit is extremely rare and only 10 such cases have been reported till date. ${ }^{[1-4]}$ These tumors are observed mostly within the first two decades of life and are usually of psammomatous variety $^{[1-3]}$ except two cases of transitional type in a 19-year-old- male and a nine-year-old female child described by Halpin et al. ${ }^{[4]}$ Authors report a unique case of transitional meningioma involving the left fronto-orbito-ethmoidal region in an adult male, which is an uncommon intradiploic meningioma in the fourth decade of life.

A 40-year-old male presented to us with complaints of insidious onset, gradually progressive, painless swelling on left side of forehead with proptosis of left eye for last eight months. Examination revealed a smooth, non-tender, bony hard swelling over left forehead, approximately $4 \times 5 \mathrm{~cm}$ in size, extending medially to the glabella and inferiorly to the left superior orbital

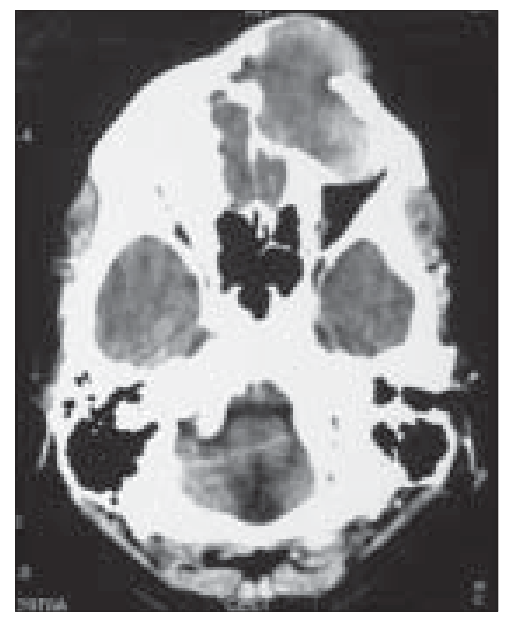

Figure 1: Contrast enhanced computerized tomography (CECT) head showing hyperdense lesion involving left orbital roof with mild contrast enhancement and expansion of left frontal and bilateral ethmoid sinuses 
margin. Computerized tomography (CT) scan revealed a hyperdense mass involving the diploe of the left orbital roof with thinning of both tables of skull causing secondary expansion of the left frontal sinus. It was also extending into bilateral ethmoid sinuses and on contrast administration, showed mild enhancement [Figure 1].

Bifrontal craniotomy was carried out. After opening the outer table of the left frontal sinus which was grossly expanded and thinned out, a well-encapsulated, firm mass was encountered. It was filling the left frontal sinus and bilateral ethmoid sinuses. The inner table was also thinned out. However, the underlying dura was not infiltrated. Periorbita of the left eye was intact. Complete excision of the mass was carried out by extradural approach. Split calvarial bone graft was used for repair of bony defect [Figure 2]. The postoperative course was

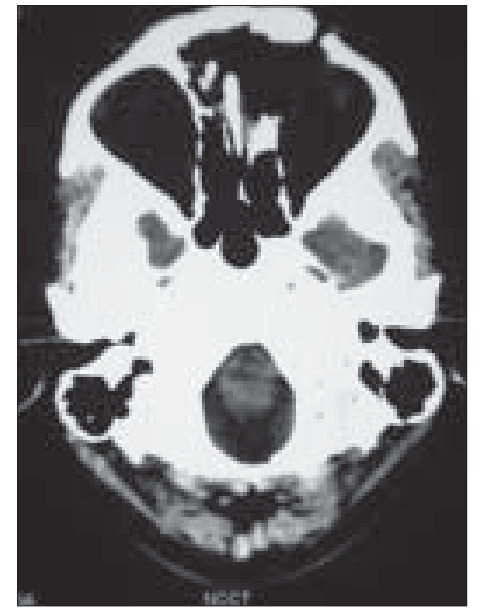

Figure 2: Postoperative non-contrast computerized tomography (NCCT) scan showing complete excision of tumor along with repair of bony defect with split calvarial bone graft

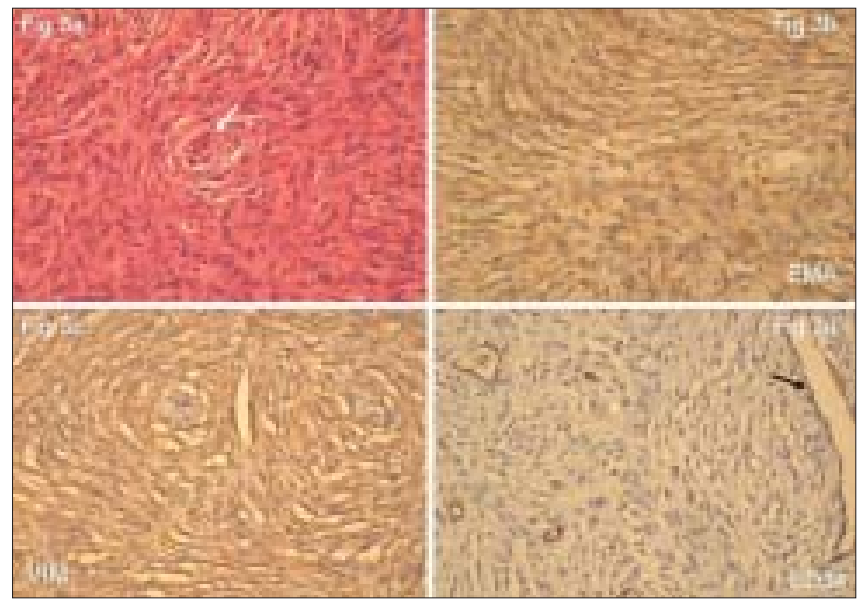

Figure 3: (a-d) Microscopy shows a cellular tumor with round to oval cells with indistinct cell outlines, minimal nuclear pleomorphism, delicate nuclear chromatin, inconspicuous nucleoli and eosinophilic cytoplasm. Cellular whorls are seen in the tumor. Fibrotic areas comprise cells with spindle-shaped nuclei. No mitosis or necrosis is present. Immunohistochemistry shows strong membrane positivity for epithelial membrane antigen (EMA) and cytoplasmic positivity for vimentin. CD34 is negative in tumor cells. The microscopic features are consistent with transitional meningioma (WHO Grade I) (x100) uneventful. He was doing well at three months follow-up and proptosis improved significantly. Histopathological features were consistent with transitional meningioma (WHO Grade I) [Figure 3].

Intradiploic meningioma is an extremely rare variety of extraneuraxial meningioma. It is postulated to arise from arachnoid cell rests captured at inappropriate sites during embryonic development or implantation of these cells by some mechanical insult like trauma or dural tear. ${ }^{[2]}$ Their origin can also be attributed to cellular dedifferentiation within the diploic space or cranial nerve sheath cells during their course through diploe..$^{[1,2,4]}$ Common presenting symptoms include exophthalmos and diplopia. ${ }^{[1]}$ Computerized tomography scan appearance of intradiploic meningioma is not typical of meningioma which enhances strongly on intravenous contrast injection. Surgery is the usual modality of treatment. Complete surgical excision is feasible in these cases because of the well-encapsulated nature of the tumor which is present in a bony shell.

Intradiploic meningioma usually does not cause dural infiltration, so extradural complete surgical excision is preferred and intradural exploration is usually not required. However, intradural exploration is advised only if there is extensive dural infiltration or dural breach is observed. The craniotomy defect can be repaired with either autologous bone graft or synthetic bone substitute. Split calvarial bone graft is an easily available cranioplasty material and is extremely useful in the repair of bony defect with better postoperative cosmetic results. It also prevents development of postoperative pulsating exophthalmos due to cranial defect. ${ }^{[2]}$

To conclude, intradiploic meningioma is an extremely rare benign skull tumor with slow progression. Complete surgical excision with appropriate cranioplasty is the optimal surgical treatment for this tumor with good cosmetic results and recovery of exophthalmos.

\section{Sachin A. Borkar, Anuj Kumar Tripathi, G. D. Satyarthee, Arvind Rishi ${ }^{1}$, S. S. Kale, B. S. Sharma}

Departments of Neurosurgery and ${ }^{1}$ Neuropathology, All India Institute of Medical Sciences, New Delhi, India. E-mail: sachin.aiims@gmail.com

\section{References}

1. Cirak B, Guven MB, Ugras S, Kutluhan A, Unal O. Fronto-orbitonasal intradiploic meningioma in a child. Pediatr Neurosurg 2000;32:48-51.

2. Desai KI, Nadkarni TD, Bhayani RD, Goel A. Intradiploic meningioma of the orbit: A case report. Neurol India 2004;52:380-2.

3. Pompili A, Caroli F, Cattani F, Iachetti M. Intradiploic meningioma of the orbital roof. Neurosurgery 1983;12:565-8.

4. Halpin SF, Britton J, Wilkins P, Uttley D. Intradiploic meningiomas: A radiological study of two cases confirmed histologically. Neuroradiology $1991 ; 33: 247-50$.

Accepted on 21-05-2008 